\title{
EXIGÊNCIAS DE FÓSFORO DISPONÍVEL PARA AVES COM O USO DO FOSFATO MONOAMÔNIO
}

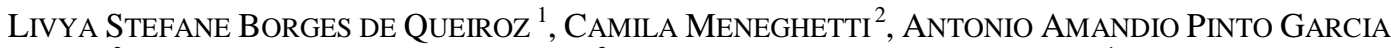

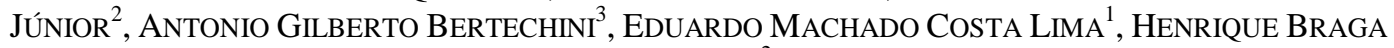 \\ OLIVEIRA $^{2}$ \\ ${ }^{1}$ Mestre em Zootecnia pela Universidade Federal de Lavras, Lavras, MG, Brasil \\ ${ }^{2}$ Pós-graduandos da Universidade Federal de Lavras, Lavras, MG, Brasil - cameneghetti@ yahoo.com.br \\ ${ }^{3}$ Professor Doutor da Universidade Federal de Lavras, Lavras, MG, Brasil
}

RESUMO

\begin{abstract}
O objetivo do trabalho foi avaliar a exigência de fósforo disponível (Pd) com o uso do fosfato monoamônio (MAP) como fonte de fósforo para frangos de corte na fase inicial (1 a 21 dias). Oitocentos pintos de corte machos de um dia de idade foram distribuídos em esquema fatorial com cinco níveis de $\mathrm{Pd}(0,28 ; 0,34 ; 0,40 ; 0,46$ e $0,52 \%)$ associados a dois níveis de cálcio (Ca) (1,00 e 0,60\%), totalizando dez tratamentos e oito repetições. Houve interação significativa entre os níveis de fósforo e cálcio da dieta, para as variáveis de desempenho, cinzas ósseas, retenção e excreção de P. Utilizando o nível de 1,0\% de
\end{abstract}

Ca na ração, os melhores resultados de desempenho e de cinzas ósseas foram obtidos quando utilizado o nível de $0,42 \%$ e $0,439 \%$ de $\mathrm{Pd}$, respectivamente. O uso de $0,60 \%$ de $\mathrm{Ca}$ proporcionou maiores teores de cinzas ósseas apenas com a utilização de $0,28 \%$ de Pd. Os maiores níveis de $\mathrm{Pd}$ proporcionaram maior excreção de $\mathrm{P}$. Ao utilizar o MAP como fonte de $\mathrm{P}$ em rações de frangos de corte na fase inicial, a utilização dos níveis de $0,43 \%$ de $\mathrm{Pd}$ e $1,00 \%$ de Ca é suficiente para promover resultados satisfatórios de desempenho e mineralização óssea.

PALAVRAS-CHAVE: desempenho; fonte de fósforo; frango de corte; mineralização óssea.

\section{AVAILABLE PHOSPHORUS FOR POULTRY WITH THE USE OF MONOAMMONIUM PHOSPHATE}

\begin{abstract}
The objective of this work was to evaluate the phosphorous requirements $(\mathrm{Pd})$ when mono-ammonium phosphate (MAP) is used as a phosphorous (P) source for broilers in the starter phase (1 to 21 days). We used 800 male chicks at one day old, in a $5 \times 2$ factorial arrangement, with five AP levels $(0.28,0.34,0.40,0.46$ and $0.52 \%)$ and two Ca levels (1.00 and 0.60), totaling ten treatments and eight replicates. Significant interaction was observed between phosphorus and calcium levels of the diet, in performance traits, bone ashes and $\mathrm{P}$ retention and
\end{abstract}

excretion. Utilizing the level of $1.0 \% \mathrm{Ca}$ in the diet, the best results of performance and bone ashes were found when the levels of $0.42 \%$ and $0.439 \% \mathrm{Pd}$ were used, respectively. Better contents of bone ashes were observed with the use of $0.60 \% \mathrm{Ca}$ in the diet only when the lowest level of $\mathrm{P}$ was used. When MAP is used as a source of $\mathrm{P}$ in the diet for broilers in the starter phase, $0.43 \%$ of $\mathrm{Pd}$ and $1.00 \%$ of $\mathrm{Ca}$ are enough to promote better performance and bone mineralization.

KEYWORDS: bone mineralization; broiler; performance; phosphorus source. 


\section{INTRODUÇÃO}

$\mathrm{O}$ elemento fósforo $(\mathrm{P})$ tem sido alvo de muitas pesquisas ao longo dos últimos anos com o enfoque na melhor forma de utilização desse mineral, tendo em vista o melhor desempenho das aves e o melhor custo/benefício. Mais recentemente, também há preocupação com a excreção e a contaminação do ambiente, havendo a necessidade de melhor conhecimento das fontes utilizadas para adequação dos níveis de $\mathrm{P}$ nas rações.

As fontes de $\mathrm{P}$ utilizadas na alimentação animal diferem não somente pela quantidade de fósforo disponível, mas também pelas matériasprimas utilizadas e pelo processamento industrial empregado na fabricação dos mesmos.

$\mathrm{O}$ fosfato monoamônio é uma fonte de $\mathrm{P}$ obtida através do tratamento da amônia com o ácido fosfórico desfluorizado ou parcialmente desfluorizado, devendo apresentar-se na forma granulada, para evitar empedramento e/ou reação do produto com outros componentes do alimento (BUTOLO, 2002). Ao contrário dos fosfatos comumente utilizados na alimentação de frangos de corte, o MAP é caracterizado por não fornecer $\mathrm{Ca}$ às dietas, implicando no aumento do nível de inclusão de calcário nas rações de frangos de corte em que se utiliza essa fonte de fósforo.

A composição do fosfato monoamônio é de aproximadamente $24 \%$ de fósforo total, $0,30 \%$ de flúor e $11 \%$ de nitrogênio não proteico, sendo que a biodisponibilidade relativa do $\mathrm{P}$ é considerada mais de $100 \%$, segundo ROSTAGNO et al. (2005).

Apesar do uso do MAP nas formulações de rações para frangos de corte, ainda existe dúvida se, ao utilizá-lo, as recomendações de Pd seriam as mesmas quando se utiliza o fosfato bicálcico como fonte. Alguns trabalhos evidenciaram a melhoria do Pd do MAP para aves, sugerindo mudanças nas recomendações quando se utiliza essa fonte de $\mathrm{P}$ (GOMES et al., 1993; DAMROM \& FLUNKER, 1991), indicando como fonte alternativa de alta biodisponibilidade. Trabalhos com poedeiras (COUTO et al., 2008) também evidenciaram melhoria da produção e peso dos ovos quando substituíram o fosfato bicálcico pelo MAP nas rações dessas aves.

Diversas fontes de $\mathrm{P}$ foram avaliadas recentemente por QUEIROZ et al. (2008) e os autores concluíram que o MAP proporciona resultados de desempenho e mineralização óssea satisfatórios, quando são utilizados níveis adequados de fósforo disponível na ração.

Além da biodisponibilidade e retenção do fósforo é extremamente importante se atentar para a relação $\mathrm{Ca} / \mathrm{Pd}$, o que pode interferir diretamente na absorção desses minerais, principalmente do fósforo. A inclusão de altos níveis de cálcio nos alimentos aumenta a necessidade de $\mathrm{P}$ para frangos de corte. $O$ cálcio interfere na absorção do $P$, complexando-o em nível de intestino, tornando-o, assim, menos disponível, além de dificultar a absorção de P fítico pela ave (DALE, 1983).

Há necessidade de constante atualização dos estudos das fontes disponíveis comercialmente e, consequentemente, dos ingredientes utilizados na alimentação animal, visto a grande importância nutricional e ambiental das fontes de $\mathrm{P}$ e o impacto no desempenho das aves.

O objetivo deste trabalho foi avaliar as exigências de Pd na fase inicial de frangos de corte (1 a 21 dias de idade) com o uso do MAP como fonte de fósforo e dois níveis de cálcio na ração.

\section{MATERIAL E MÉTODOS}

Os experimentos (desempenho e metabolismo) foram conduzidos no Setor de Avicultura do Departamento de Zootecnia da Universidade Federal de Lavras (UFLA), Minas Gerais, sendo utilizados 800 pintos de corte machos Cobb-500 de um dia de idade, com peso médio de $42 \pm 2,0 \mathrm{~g}$, distribuídos em gaiolas de metabolismo confeccionadas em arame galvanizado, em ambiente com controle de temperatura e ventilação. Utilizou-se delineamento experimental inteiramente casualizado, em esquema fatorial $(5 \times 2)$, totalizando 10 tratamentos, com oito repetições de 10 aves por unidade experimental. Foram estudados cinco níveis de Pd $(0,28 ; 0,34 ; 0,40 ; 0,46$ e $0,52 \%)$ e dois níveis de $\mathrm{Ca}(0,60$ e $1,00 \%)$. As relações de $\mathrm{Ca} / \mathrm{Pd}$ foram: 2,$1 ; 1,7 ; 1,5 ; 1,3 ; 1,1$ para $0,60 \%$ de $\mathrm{Ca}$ e 3,$5 ; 2,9$; 2,$5 ; 2,1 ; 1,9$ para $1,00 \%$.

As rações experimentais (Tabela 1) foram à base de milho e farelo de soja, formuladas de acordo com as recomendações de ROSTAGNO et al. (2005) para a fase de 1 a 21 dias de idade das aves, com exceção dos níveis de $\mathrm{Ca}$ e $\mathrm{Pd}$, que estão descritos na Tabela 2. 
TABELA 1. Composição percentual das rações experimentais e níveis nutricionais calculados (exceto Ca e P)

\begin{tabular}{|c|c|}
\hline Ingredientes & $\%$ \\
\hline Milho & 53,71 \\
\hline Farelo de Soja & 37,10 \\
\hline Óleo de Soja & 3,950 \\
\hline Sal & 0,460 \\
\hline Suplemento Mineral ${ }^{1}$ & 0,100 \\
\hline Suplemento Vitamínico ${ }^{2}$ & 0,100 \\
\hline DL-Metionina - $99 \%$ & 0,255 \\
\hline L-Lisina $-78 \%$ & 0,091 \\
\hline L-Treonina $-98 \%$ & 0,023 \\
\hline Anticoccidiano $^{3}$ & 0,040 \\
\hline Antibiótico ${ }^{4}$ & 0,025 \\
\hline Cloreto de Colina $-70 \%$ & 0,057 \\
\hline \multicolumn{2}{|l|}{ Composição Calculada } \\
\hline Energia Metabolizável (kcal/kg) & 3000 \\
\hline Proteína Bruta $(\%)$ & 21,5 \\
\hline Lisina dig. $(\%)$ & 1,13 \\
\hline Metionina dig $(\%)$ & 0,552 \\
\hline Metionina + Cistina dig $(\%)$ & 0,840 \\
\hline Sódio $(\%)$ & 0,200 \\
\hline \multicolumn{2}{|c|}{ Fornecimento por kg de ração: $60 \mathrm{mg} \mathrm{Zn;} 30 \mathrm{mg} \mathrm{Fe} ; 9 \mathrm{mg} \mathrm{Cu} ; 60 \mathrm{mg} \mathrm{Mn;} 1,0 \mathrm{mg} \mathrm{I}}$. \\
\hline \multicolumn{2}{|c|}{$\begin{array}{l}2 \text { Fornecimento por kg de ração12.000 UI vit. A; } 2.200 \mathrm{UI} \text { vit D3; } 30 \mathrm{mg} \text { vit. E; } 2,5 \mathrm{mg} \text { Vit. } \mathrm{K} 3 ; 2,2 \mathrm{mg} \text { vit.B1; } 6 \mathrm{mg} \text { vit. B2; } 3,3 \mathrm{mg} \\
\text { vit. B6; } 16 \text { g vit. B12; 0,11 mg biotina; } 1,0 \mathrm{mg} \text { ácido fólico; } 13,0 \mathrm{mg} \text { ácido pantotênico; } 53 \mathrm{mg} \text { nicotinamida; } 120 \mathrm{mg} \text { antioxidante } \\
\text { BHT. }\end{array}$} \\
\hline
\end{tabular}

TABELA 2. Composição percentual das rações experimentais referente ao fosfato monoamônio (MAP), calcário calcítico e caulim

\begin{tabular}{|c|c|c|c|c|c|c|}
\hline \multirow[b]{2}{*}{ Ingredientes } & $\% \mathrm{Pd}$ & 0,28 & 0,34 & 0,40 & 0,46 & 0,52 \\
\hline & \multicolumn{6}{|c|}{ Rações $(0,60 \% \mathrm{Ca})$} \\
\hline MAP & & 0,728 & 0,984 & 1,240 & 1,496 & 1,752 \\
\hline Calcário Calcítico & & 1,218 & 1,218 & 1,218 & 1,218 & 1,218 \\
\hline \multirow[t]{2}{*}{ Caulim } & & 2,126 & 1,870 & 1,614 & 1,358 & 1,102 \\
\hline & \multicolumn{6}{|c|}{ Rações $(1,00 \% \mathrm{Ca})$} \\
\hline MAP & & 0,728 & 0,984 & 1,240 & 1,496 & 1,752 \\
\hline Calcário Calcítico & & 2,234 & 2,234 & 2,234 & 2,234 & 2,234 \\
\hline Caulim & & 1,109 & 0,853 & 0,597 & 0,341 & 0,085 \\
\hline
\end{tabular}

O desempenho das aves foi avaliado pelas características de ganho de peso, consumo de ração e conversão alimentar, no período de 1 a 19 dias de idade, e para a determinação do balanço de fósforo foram utilizadas as mesmas rações e aves em ensaio metabólico, durante três dias $\left(20^{\circ}\right.$ ao $22^{\circ}$ dia de idade das aves), com o método tradicional de coleta de excretas.

Para o início do ensaio de metabolismo, foram realizadas as pesagens das aves e da ração 
fornecida. A ração foi marcada com óxido férrico para sinalizar o início da coleta. Foram realizadas duas coletas diárias, sendo uma às 8:00 e a outra às 15:00 horas, evitando, assim, o início de processo fermentativo nas excretas. Durante a coleta, foram retirados resíduos de ração e de penas. As amostras de excretas foram homogeneizadas, pré-secas, queimadas a $550^{\circ} \mathrm{C}$ e solubilizadas, sendo determinados os coeficientes de retenção de fósforo (CRP) e a excreção de $\mathrm{P}$ total $(\mathrm{Pt})$, em matéria seca, pelas fórmulas abaixo.

$$
\mathrm{CRP}=\frac{\text { consumo de } \mathrm{P}(\mathrm{g})-\text { excreçãode } \mathrm{P}(\mathrm{g})}{\text { consumo de } \mathrm{P}(\mathrm{g})} \times 100
$$

Excreção de Pt $=\frac{\text { quantidade de excreta }(\mathrm{g}) \times \% \text { Pt na excreta }}{100}$

As análises de fósforo das excretas seguiram o método da AOAC (1990), através de colorimetria, com a leitura realizada em espectrofotômetro a 400 nm.

Ao final do período experimental $\left(23^{\circ}\right.$ dia $)$, foram abatidas duas aves por parcela, sendo retiradas as tíbias, para a determinação das cinzas ósseas. As tíbias foram desengorduradas inicialmente utilizando-se recipiente fechado contendo éter etílico por dois dias. Em seguida, foram lavadas com éter etílico por 12 horas em aparelho Soxhlet. Após secagem em estufa a $105^{\circ} \mathrm{C}$ foram submetidas à incineração à temperatura de $550^{\circ} \mathrm{C}$ por aproximadamente 8 horas, obtendo-se, dessa forma, as cinzas na matéria seca desengordurada.
Todas as análises foram realizadas no Laboratório de Pesquisa Animal do Departamento de Zootecnia da UFLA.

As temperaturas máxima e mínima foram registradas diariamente, às 16 horas, sendo observadas as médias de 29,5 e $22,5^{\circ} \mathrm{C}$, respectivamente. $\mathrm{O}$ programa de iluminação adotado foi o de $24 \mathrm{~h}$ de luz constante durante o ensaio.

As análises estatísticas foram realizadas utilizando-se o pacote computacional SISVAR, descrito por FERREIRA (2000), por meio da análise de regressão para os níveis de fósforo e teste $\mathrm{F}$ para os níveis de cálcio, a 5\% de probabilidade.

\section{RESULTADOS E DISCUSSÃO}

Os resultados referentes ao desempenho de frangos de corte na fase de 1-19 dias de idade estão apresentados na Tabela 3, sendo verificada interação significativa $(\mathrm{P}<0,05)$ entre os níveis de cálcio e fósforo para as variáveis de desempenho dos frangos de corte. Para o nível dietético de Ca de 1,00\%, houve maiores valores de consumo de ração e de ganho de peso, com os níveis de $\mathrm{Pd}$ de 0,419 e $0,426 \%$, respectivamente, utilizando o efeito quadrático observado. Ao utilizar o fosfato bicálcico como fonte de fósforo, RUNHO et al. (2001) sugeriram o valor de $0,455 \%$ de Pd para frangos de corte de 1 a 21 dias de idade. Segundo ROSTAGNO et al. (2005), a exigência nutricional de Pd para frangos de corte é de $0,466 \%$ Pd de 1 a 7 dias e de $0,439 \%$ Pd de 8 a 21 dias de idade das aves.

TABELA 3. Consumo de ração (CR), ganho de peso (GP) e conversão alimentar (CA) de frangos de corte machos de 1 a 19 dias de idade alimentados com rações contendo diferentes níveis de Pd com dois níveis de $\mathrm{Ca}$

\begin{tabular}{lccccccc}
\hline \multicolumn{7}{c}{ Consumo de Ração } \\
\hline \multirow{2}{*}{ Nível de Ca $(\%)$} & 0,28 & 0,34 & 0,40 & 0,46 & 0,52 & Média & CV (\%) \\
\cline { 2 - 8 } & $926 \mathrm{~A}$ & $941 \mathrm{~A}$ & $916 \mathrm{~B}$ & $915 \mathrm{~B}$ & $902 \mathrm{~A}$ & 920 & 2,59 \\
1,00 & $855 \mathrm{~B}$ & $937 \mathrm{~A}$ & $946 \mathrm{~A}$ & $946 \mathrm{~A}$ & $907 \mathrm{~A}$ & 918 & \\
Média & 891 & 939 & 931 & 931 & 905 & 919 & \\
\hline \multicolumn{7}{c}{ Ganho de Peso } \\
\hline 0,60 & $697 \mathrm{~A}$ & $698 \mathrm{~A}$ & $672 \mathrm{~B}$ & $682 \mathrm{~B}$ & $653 \mathrm{~B}$ & 680 & \multirow{2}{*}{2,73} \\
1,00 & $631 \mathrm{~B}$ & $684 \mathrm{~A}$ & $717 \mathrm{~A}$ & $706 \mathrm{~A}$ & $681 \mathrm{~A}$ & 684 & \\
Média & 664 & 691 & 695 & 694 & 667 & 682 & \\
\hline \multicolumn{7}{c}{ Conversão Alimentar ${ }^{1}$} \\
\hline 0,60 & $1,33 \mathrm{~A}$ & $1,35 \mathrm{~A}$ & $1,36 \mathrm{~B}$ & $1,34 \mathrm{~A}$ & $1,38 \mathrm{~B}$ & 1,35 & \multirow{2}{*}{2,47} \\
1,00 & $1,36 \mathrm{~A}$ & $1,37 \mathrm{~A}$ & $1,32 \mathrm{~A}$ & $1,34 \mathrm{~A}$ & $1,33 \mathrm{~A}$ & 1,35 & \\
Média & 1,35 & 1,36 & 1,34 & 1,34 & 1,36 & 1,35 & \\
\hline
\end{tabular}

${ }^{1}$ Interação significativa $(\mathrm{P}<0,05)$ : letras maiúsculas com médias diferentes na coluna diferem estatisticamente pelo teste de $\mathrm{F}$. Para $0,6 \%$ de Ca: $\mathrm{CR}=969,11-123,20 x, \mathrm{r}^{2}=65,24 \%$; $\mathrm{GP}=749,94-173,33 \mathrm{x}, \mathrm{r}^{2}=77,09 \%$; $\mathrm{CA}=1,28+0,18 \mathrm{x}, \mathrm{r}^{2}=62,22 \%$ e para $1,00 \%$ de Ca: $C R=85,23+4154,36 x-4956,20 x^{2}\left(R^{2}=95,88 \%\right) ; G P=1,74+3348,90 x-3932,29 x^{2}\left(R^{2}=98,98 \%\right)$. 
Estes valores de Pd encontrados na literatura foram obtidos utilizando-se o fosfato bicálcico como fonte de fósforo e pôde-se verificar que os valores de Pd indicados para essa fase são maiores dos que o obtidos neste trabalho, portanto, vindo ao encontro dos objetivos de redução da suplementação e excreção deste elemento. Já para o nível de $\mathrm{Ca}$ de $0,6 \%$, houve efeito linear $(\mathrm{P}<0,05)$ dos níveis dietéticos de Pd havendo redução no consumo e ganho de peso e piora na conversão alimentar.

Considerando os níveis de Ca em cada nível de Pd estudado, verificaram-se resultados superiores de desempenho para o menor nível de $\mathrm{Ca}$ apenas no menor nível de $\mathrm{Pd}$, havendo inversão do comportamento com o aumento do Pd na ração, em que maiores valores puderam ser observados com o uso de $1,00 \%$ de $\mathrm{Ca}$ na ração. Esses resultados demonstraram o efeito da adição desses minerais na dieta sobre a manutenção do equilíbrio orgânico por meio da relação $\mathrm{Ca} / \mathrm{P}$. Esses minerais estão intimamente associados no metabolismo animal, muitas vezes combinados entre si, de modo que a carência de um deles na dieta limita o desempenho das aves (MACARI et al., 2002).

SHAFEY et al. (1991) constataram que o excesso de fósforo e de cálcio na dieta pode reduzir o desempenho das aves, por meio da diminuição na digestibilidade de nutrientes, em função da queda da absorção dos mesmos e da permeabilidade da parede intestinal. Além disso, o excesso de cálcio e fósforo na dieta pode resultar em redução na disponibilidade desses minerais pela formação de fosfatos de cálcio, insolúvel no trato digestível, o que, consequentemente, reduz a absorção de ambos.

Os resultados de cinzas ósseas das aves (Tabela 4) mostram interação significativa $(\mathrm{P}<0,01)$ dos níveis de $\mathrm{Ca}$ e Pd. Ao se utilizar o nível de $1,00 \%$ de $\mathrm{Ca}$, houve efeito quadrático $(\mathrm{P}<0,01)$ dos níveis de Pd, indicando o valor de 0,439\% de Pd como responsável pelos maiores teores de cinzas ósseas nas tíbias. Ao se comparar os níveis de cálcio $(0,60$ e $1,00 \%)$ dentro de um mesmo nível de Pd, pode-se observar maiores valores de cinzas para o menor nível de cálcio $(0,60 \%)$ apenas para os tratamentos com nível inferior de Pd $(0,28 \%)$.

TABELA 4. Valores de cinzas ósseas (CO) das tíbias de frangos de corte machos de 1 a 21 dias de idade alimentados com rações contendo diferentes níveis de $\mathrm{Pd}$ com dois níveis de $\mathrm{Ca}$

\begin{tabular}{lccccc}
\hline \multirow{2}{*}{ Nível de $\mathrm{Ca}(\%)$} & \multicolumn{5}{c}{${\text { Nível de Pd }(\%)^{1}}^{1}$} \\
\cline { 2 - 6 } & 0,28 & 0,34 & 0,40 & 0,46 & 0,52 \\
\hline 0,60 & $50,35 \mathrm{~A}$ & $50,30 \mathrm{~B}$ & $50,90 \mathrm{~B}$ & $50,61 \mathrm{~B}$ & $49,85 \mathrm{~B}$ \\
1,00 & $48,69 \mathrm{~B}$ & $52,05 \mathrm{~A}$ & $52,84 \mathrm{~A}$ & $52,10 \mathrm{~A}$ & $52,33 \mathrm{~A}$ \\
\hline $\mathrm{CV}(\%)$ & 2,42 & \\
\multicolumn{1}{l}{ Interação significativa $(\mathrm{P}<0,05) \mathrm{Ca} \times$ Pd: letras maiúsculas com médias diferentes na coluna diferem estatisticamente pelo teste de $\mathrm{F}$} \\
(P<0,05). Para 1,00 \% de Ca: $\mathrm{CO}=23,05+136,14 \mathrm{x}-154,91 \mathrm{x}^{2}, \mathrm{r}^{2}=88,44 \%$.
\end{tabular}

Os maiores valores de cinzas ósseas foram observados quando se obteve a relação de $\mathrm{Ca} / \mathrm{Pd}$ em, aproximadamente, 2:1. Isso está de acordo com os resultados obtidos no ensaio de desempenho, em que houve melhores resultados ao se utilizar o nível de $1,00 \%$ de Ca nos maiores níveis de Pd. Porém, foi observado maior ganho de peso das aves submetidas às rações com $0,60 \%$ de $\mathrm{Ca}$ com o menor nível de $\mathrm{Pd}$ $(0,28 \%)$.

Uma relação linear entre a concentração de fósforo na dieta de aves e valores de mineralização óssea puderam ser observados por CORTELAZZI (2006), por meio do aumento de $36 \%$ nas cinzas das tíbias de frangos de corte, em relação à dieta basal, na fase de crescimento, com a adição de $0,16 \%$ de $\mathrm{P}$ em relação à suplementação de $0,08 \%$ de $\mathrm{P}$ à dieta.

Cinzas ósseas tem sido o método mais utilizado para determinação da disponibilidade biológica de fósforo em aves (WALDROUP, 1996). $\mathrm{O}$ método, apesar de ser dispendioso em relação à necessidade de tempo e mão-de-obra, é um dos mais precisos para essa finalidade (CORTELAZZI, 2006).

$\mathrm{Na}$ Tabela 5 encontram-se os resultados de retenção e excreção de fósforo em que pode ser observada interação significativa $(\mathrm{P}<0,01)$ dos níveis de $\mathrm{Ca}$ e $\mathrm{Pd}$ avaliados, para a excreção de Ptotal (g/dia) e para os CRP (\%). 
TABELA 5. Coeficientes de retenção (\%) e excreção (g/dia) de fósforo para frangos de corte de 1 a 21 dias submetidos as rações contendo níveis crescentes de Pd e dois níveis de $\mathrm{Ca}$

\begin{tabular}{|c|c|c|c|c|c|c|}
\hline \multicolumn{7}{|c|}{ Coeficiente de Retenção de Fósforo (CRP) ${ }^{1}$} \\
\hline \multirow{2}{*}{$\begin{array}{l}\text { Nível de Ca } \\
\text { (\%) }\end{array}$} & \multicolumn{6}{|c|}{ Nível de Pd (\%) } \\
\hline & 0,28 & 0,34 & 0,40 & 0,46 & 0,52 & $\mathrm{CV}(\%)$ \\
\hline 0,60 & $55,99 \mathrm{~B}$ & 53,53B & $52,54 \mathrm{~A}$ & $52,76 \mathrm{~A}$ & $53,67 \mathrm{~A}$ & \multirow{2}{*}{5,79} \\
\hline 1,00 & $66,05 \mathrm{~A}$ & $60,53 \mathrm{~A}$ & $53,15 \mathrm{~A}$ & $49,37 \mathrm{~A}$ & $46,70 \mathrm{~B}$ & \\
\hline \multicolumn{7}{|c|}{ Excreção de Pt (g/dia) ${ }^{1}$} \\
\hline 0,60 & $0,24 \mathrm{~B}$ & $0,28 \mathrm{~B}$ & $0,30 \mathrm{~A}$ & $0,31 \mathrm{~A}$ & $0,33 \mathrm{~A}$ & \multirow[b]{2}{*}{7,63} \\
\hline 1,00 & $0,18 \mathrm{~A}$ & $0,24 \mathrm{~A}$ & $0,30 \mathrm{~A}$ & $0,35 \mathrm{~B}$ & $0,41 \mathrm{~B}$ & \\
\hline
\end{tabular}

${ }^{1}$ Interação significativa: letras maiúsculas com médias diferentes na coluna diferem estatisticamente pelo teste $\mathrm{F}(\mathrm{P}<0,05)$. Para $0,60 \%$ Ca: Excreção de $\mathrm{Pt}=0,14+0,38 x, \mathrm{r}^{2}=92,85 \%$ e para 1,0\% Ca: CRP= 88,39 - 83,08x, $\mathrm{r}^{2}=96,89 \%$; Excreção de $\mathrm{Pt}=-0,093+0,967 \mathrm{x}$, $r^{2}=99,90 \%$.

Maiores CRP foram encontrados para o nível de $1,0 \%$ de $\mathrm{Ca}$, com a utilização de níveis menores de $\mathrm{Pd}$ na ração $(0,28$ e $0,34 \%)$. Para o menor nível de Ca utilizado $(0,60 \%)$, valores superiores de CRP foram obtidos para o tratamento com $0,52 \%$ de $\mathrm{Pd}$. Ao utilizar o nível de $1,00 \%$ de $\mathrm{Ca}$ na dieta, observou-se uma regressão linear $(\mathrm{P}<0,05)$ dos níveis de Pd avaliados, em que o menor nível de Pd $(0,28 \%)$ foi responsável pelo maior CRP $(66,05 \%)$.

A utilização de menores níveis de Pd, com as dietas contendo $1,00 \%$ de $\mathrm{Ca}$, foi responsável por menores excreções de Pt. Os resultados apresentaram comportamento contrário ao se utilizar o nível de $0,6 \%$ de $\mathrm{Ca}$, em que se pôde observar uma menor excreção de Ptotal para o maior nível de Pd $(0,52 \%)$. A análise de regressão indicou efeito linear dos níveis de $\mathrm{Pd}$, e, independentemente do teor de $\mathrm{Ca}$ utilizado na ração, a utilização de menores níveis de $\mathrm{P}$ foi responsável pela menor excreção do mesmo.

Os resultados obtidos demonstraram que, ao utilizar níveis de $\mathrm{Pd}$ próximos aos recomendados pela literatura $(0,40$ e $0,46 \%)$, os níveis de cálcio estudados não influenciaram a retenção de $\mathrm{P}$ de frangos de corte aos 21 dias de idade. Da mesma forma, não houve diferença entre os níveis de cálcio sobre a excreção de $\mathrm{P}$ para o nível de $0,40 \%$ de $\mathrm{Pd}$.

Ao avaliar o efeito da restrição de $\mathrm{Ca}$ e $\mathrm{P}$ em frangos de corte de 1 a 18 dias, YAN et al. (2005), observaram maior absorção para as aves que receberam dietas com níveis inferiores desses minerais $(0,30 \% \mathrm{Pd}$ e $0,60 \% \mathrm{Ca})$ com relação $2: 1$ de $\mathrm{Ca} / \mathrm{P}$. O metabolismo desses minerais envolve um controle de autorregulação, demonstrado pela capacidade adaptativa das aves quando expostas a situações de restrição de nutrientes, por meio do aumento da taxa de absorção e eficiência de utilização, com queda na excreção dos nutrientes.

\section{CONCLUSÃO}

Utilizando-se o MAP como fonte suplementar de fósforo e o nível de 1,00\% de $\mathrm{Ca}$, o nível ideal de Pd das rações de frangos de corte na fase de 1 a 21 dias de idade das aves é de 0,43\%, otimizando as características de desempenho e favorecendo a mineralização óssea

\section{REFERÊNCIAS}

ASSOCIATION OF OFFICIAL ANALYTICAL CHEMISTS - AOAC. Official methods of analysis, 15. ed. Washington D.C., 1990. 1141 p.

BRASIL. Ministério da Agricultura e Reforma Agraria. Normais climatologicas: 1961 - 1990. Brasilia: MARA, 1992. $84 \mathrm{p}$.

CORTELAZZI, C.Q.L. Fósforo disponível para frangos de corte em fosfatos para alimentação animal. 2006.62 p. Dissertação (Mestrado em Medicina Veterinária) Universidade de São Paulo, Pirassununga, SP. Disponível em

http://www.teses.usp.br/teses/disponiveis/10/10135/tde27042007-140226/pt-br.php

COUTO, H.P.; NERY, V.L.H.; FONSECA, J.B.; CHIQUIERI, J.; CARNEIRO, L.C.R.; LOMBARDI, C. T. Fontes alternativas de cálcio e fósforo para poedeiras comerciais. Revista Brasileira de Zootecnia, v.37, n.8, p.1419-1423, 2008.

DAMRON, B.L.; FLUNKER, L.K. Supplementation of broilers drinking water with liquid ammonium polyphosphate. British Poultry Science, v.32, n.2, p.377382, 1991

DALE, N. Necessidades de fósforos para pollos. Avicultura Professional, p.80-83, 1983.

FERREIRA, D. F. Sistema de análises de variância para dados balanceados. Lavras: UFLA, 2000. (SISVAR 
4. 1. - pacote computacional).

MACARI, M.; FURLAN, R.L.; GONZALES, L. Fisiologia aviária aplicada a frangos de corte. Jaboticabal, FUNEP/UNESP, 2002. 375 p.

QUEIROZ, L.S.; BERTECHINI, A.G.; RODRIGUES, P.B.; GUERREIRO, M.C. Utilização de fosfatos comerciais para frangos de corte na fase inicial. Pesquisa Agropecuária Brasileira, v.43, n.10, p.1421-1427, 2008.

ROSTAGNO, H.S.; ALBINO, L.F.T.; DONZELE, J.L.; GOMES, P.C.; OLIVEIRA, R.F.; LOPES, D.C.; FERREIRA, A.S.; BARRETO, S.L.T. Tabelas Brasileiras para Aves e Suínos. Composição de alimentos e exigências nutricionais. Viçosa, MG, UFV, 2005, 186p.

RUNHO, R.C.; GOMES, P.C.; ROSTAGNO, H.S.; ALBINO, L.F.T.; LOPES, P.S.; POZZA, P.C. Exigência de fósforo disponível para frangos de corte machos e fêmeas de 1 a 21 dias de idade. Revista Brasileira de Zootecnia, v.30, p.187-196, 2001.

SHAFEY, T.M., McDONALD M.W., DINGLE J.G. Effects of dietary calcium and avaliable phosphorus concentration on digesta $\mathrm{ph}$ and on the avaliable of calcium, iron, magnesium and zinc from the intestinal contents of meat chickens. British Poultry Science, v.32, p.185-194, 1991.

WALDROUP, P.W. Bioassays remain necessary to estimate phosphorous, calcium bioavailability. Feedstuffs, v.68, n.4, p.13-23, 1996.

YAN, F.; ANGEL, R.; ASHWELL, C.; MITCHELL, A.; CHRISTMAN, M. Evaluation of the Broiler's Ability to Adapt to an Early Moderate Deficiency of Phosphorus and Calcium. Poultry Science, v.84, p.1232-1241, 2005. 